\title{
Human gait-based gender classification using neutral and non-neutral gait sequences
}

Clasificación de género basada en la marcha humana usando secuencias de marcha neutrales y no neutrales

Author:

Zhyar Q. Mawlood ${ }^{1}$

Azhin T. Sabir ${ }^{2}$

\section{SCIENTIFIC RESEARCH}

How to cite this paper:

Mawlood. Z. Q. and Sabir. A.T Human gaitbased gender classification using neutral and non-neutral gait sequences. Innovaciencia. 2019; 7 (1): 1-13.

http://dx.doi.org/10.15649/2346075X.689

Reception date:

Received: 22 April 2018

Accepted: 10 June 2018

Published: 28 December 2018

Keywords:

Gait; Gait Entropy Energy Image; k-NN; SVM

\section{ABSTRACT}

A biometric system offers automatic identification of an individual based on characteristic possessed by the individual. Biometric identification systems are often categorized as physiological or behavioural characteristics. Gait as one of the behavioural biometric recognition aims to recognize an individual by the way he/she walk. In this paper we propose gender classification based on human gait features using wavelet transform and investigates the problem of non-neutral gait sequences; Coat Wearing and carrying bag condition as addition to the neutral gait sequences. We shall investigate a new set of feature that generated based on the Gait Energy Image and Gait Entropy Image called Gait Entropy Energy Image (GEnEI). Three different feature sets constructed from GEnEI based on wavelet transform called, Approximation coefficient Gait Entropy Energy Image, Vertical coefficient Gait Entropy Energy Image and Approximation \& Vertical coefficients Gait Entropy Energy Image Finally two different classification methods are used to test the performance of the proposed method separately, called k-nearest-neighbour and Support Vector Machine. Our tests are based on a large number of experiments using a well-known gait database called CASIA B gait database, includes 124 subjects (93 males and 31 females). The experimental result indicates that the proposed method provides significant results and outperform the state of the art.

Zhyar Q. Mawlood, Computer department, College of engineering, Al-kitab University College, Altin copre, kirkuk, Iraq - .

Azhin T. Sabir, Department of Software Engineering, FENG, Koya University, Koya, Iraq, azhin.sabir@koyauniversity.org, 


\section{INTRODUCTION}

Individual recognition is a necessary task to identify/ verify human. Biometrics aim to measures and analyzes individual's characteristics for the purpose of identification/ verification. Biometric comes from the Greek words contain two words bios (life), and metric (measure). The meaning of biometric system in today's world has been maintained by the must do a large-scale identity management system whose functionality relies on the accurate determination of an individual's identity: ID cards, punch, a secret password, and PIN are used for personal identification. Biometric system, are basically classified into two parts; physiological and behavioural characteristics ${ }^{(1)}$. Physiological features are connected to the shape of the body, like a fingerprint, palm veins, face recognition. A physiological biometric might distinguish by one's voice, DNA, handprint or behaviour. Conversely, behavioural features are connected to the pattern of behaviour of a person, including gait, handwritten signature, and voice. Behavioural features can be recognized by identification systems.

Gait as a type of behavioural characteristics recently has been gained a great interest by the researcher. To use gait as a biometric recognition/identification system is strongly motivated by the need for an automated identification system for monitoring applications and visual surveillance. Generally, we can divide gait analysis into two parts: Gait recognition where identify subject's ID and gait classification which includes gender classification, action classification, and estimation of age $\stackrel{(2)}{\text {. }}$

There are many types of research related to human gait recognition $\stackrel{(3)}{(4)}$, but only a few recent works

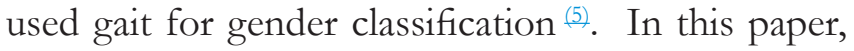
we propose gender classification based on gait feature using neutral and non-neutral gait sequence.

\section{RELATED WORK}

Gait is one of the behavioral biometric traits that can play a major role in security surveillance and authentication applications. Basically, gait signature can be used for two main purposes; human identification, where identifying the subject's ID in the spe- cific environment and human classification, including gender classification ${ }^{(6)}$, action classification, and estimation of age. Gender classification approaches plays an important role in many applications, especially in security surveillance systems. In (7) a method proposed to study gender classification from human walking manner, they demonstrated that observers are able to recognize the point-light walkers' gender.

Gait features are divided into two main categories: model-based and motion-based. Model-based gait recognition approaches converge on improving the structural model of human motion, and the gait patterns are then generated from the model parameters or tracking body components such as limbs, legs, arms, and thighs ${ }^{(8)}(9)$ and ${ }^{(10)}$. In ${ }^{(8)}$ the model-based gender classification method proposed using gait feature. The dataset created based on Kinect sensor; 30 balanced number of participants recorded from male and female. Dynamic features of several limbs were extracted and represented by Euler angles in coordinate system attached to the human body, then principal components analysis (PCA) was used for reducing the dimension of the feature vectors, and non-linear SVM with RBF kernel used to classify hu-

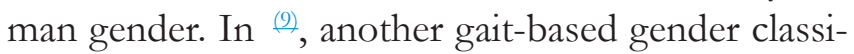
fication scheme suggested based on anthropometric and gait attributes using data recorded by Kinect sensor; two sets of features were extracted: the first set is spatiotemporal, with parameters calculated using the average step length of gait cycles; the second set is Kinematic parameters were extracted from the angles' curves created in one gait cycle. k-NN, SVM and Multilayer Perceptron (MLP) are used as classification methods. In ${ }^{(10)}$ two sets of gait features extracted to be used for gender classification: the first is based on height and the length of arms and legs, called physical features; the other set is a set of dynamic features, (like; the distance between left and right elbows, knees, hands, and ankles), then C-SVM, and V-SVM are used as classification methods.

Conversely, motion-based (Model-free) recognition approaches describe the motion pattern of the body, regardless of its fundamental structure $\stackrel{(11)}{,} \stackrel{(12)}{ }$ and ${ }^{(13)}$. In ${ }^{(11)}$ static features are used that describe the silhouette appearance of a human walking for person identification and gender recognition. To represent 
appearance, the human silhouettes have been divided into seven regions that could be fitted with ellipses, movement and some parameters of the ellipses model of the same region were performed across all the frames of the sequences, which is resulting in a set of 57 attributes at per sequence. The classification experiments on the MIT Gait Database led to an accuracy close to $80 \%$.

Spatial-temporal silhouette analysis is suggested in $\stackrel{(12)}{ }$, in their proposed method, Eigenspace transformation based on the Principal component analysis (PCA) is used to reduce the dimensionality of the image sequence and supervised algorithm is used for gait classification. In ${ }^{(13)}$ another method proposed that deale with a different appearance-based method for gait-based gender recognition, the method was tested on the CASIA gait database. Given a sequence of gait silhouettes, a Gait Energy Image (GEI) is created. The GEI is divided into 5 regions, head/hairstyle, chest, back, waist/buttocks and legs, which are weighted as regards a previous psychological study. Experiments involved a single subset composed of 31 women and 31 randomly selected men that fed a Support Vector Machine (SVM) with a linear kernel. The best classification result was an accuracy of $95.97 \%$. Nevertheless, the use of only one subset raises doubts about the reliability of the result.

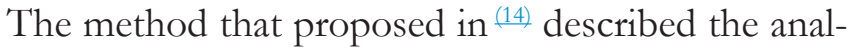
ysis of body using human radiation frequency gender classification. The human radiation frequency is experimentally studied from 33 healthy humans. They used 17 males and 16 females and k-Nearest Neighbor (k-NN) as a classifier is employed for gender classification. They first analysed raw data set and post-processing dataset and then they compare their classification results. They obtained accuracy of $93.8 \%$.

Human gait challenges are divided into two factors: External and Internal factor; clothes, viewing angles and objects carried are classified as an external factor, while drunkenness, aging, gaining or losing weight and pregnancy are classified as internal factors.

The authors of ${ }^{(15)}$ and ${ }^{(16)}$ addressed external factor challenges by investigating the problem of two variations: clothing (coats wearing) and carrying bag con- dition in addition to the normal gait sequence. In $\stackrel{(15)}{-}$ a hierarchy approach proposed for gender classification based on human gait. In this method, different influence factors like clothing and carrying condition changes are used. they used 31-fold cross validation is employed to evaluate the CCR, moreover, low dimensional discriminative representation obtained as the Gabor-MMI feature and gender-related Gaussian Mixture Model with Hidden Markov Models (GMM-HMMs) are constructed in this classification model.

In $\frac{(16)}{1}$ three different sets of the feature are proposed based on wavelet transform the first called Spatio-temporal distance that extracted based on thedistance of the human body parts (like feet, knees, hand, Human Height and shoulder). The other two sets of feature extracted based on LL and non-LL subbands. This method used 10 -fold cross validation followed by k-NN which is used as a classification method.

In this paper we aim to propose gait-based gender classification and we will address the same challeges as investigated in ${ }^{(15)}$ and ${ }^{(16)}$ (carrying bag and coat wearing gait sequences as addition to using neutral (normal) gait sequences), Moreover, a new feature set will be generated base on GEI and Gait Entropy Image (GEnI) called GEnEI.

\section{PROPOSED METHOD}

Gait-based gender classification is designed to detect human gender based on his/her gait. In this method, we address three types of gait sequences; Neutral $(\mathrm{Nu})$, Coat Wearing $(\mathrm{CW})$ and Carrying Bag (CB) gait sequences. The method has been developed based on three main steps (see Figure 1). The first step is the pre-processing, this is starting by processing all the videos from CASIA database, each video is containing a single individual walking in a certain view direction. The selected viewing direction in our proposed method is 90 degrees (i.e. side view), which provide more dynamic information about human gait. The videos are pre-processed by applying a background subtraction method to generate the silhouettes. In this paper, we used frame difference method by calculating the difference between the 
consecutive frame and frame reference. To generate the frame reference we find the average of the first ten frame assuming that there is no object on the scene. Box boundary, Normalization and alignment are applied for each of the silhouette images, this is followed by the gait cycle estimation as a final process in this step.

The second step is the process of feature extraction, gait feature is generated based on wavelet transform and GEnEI. In this method three feature vectors have been created; the first feature vector extracted from LL2 wavelet subband, by applying GEnEI method on the sequence of frames during one gait cycle and called Approximation Coefficient Gait Entropy Energy Image (AGEnEI). The second feature vector extracted from LH2 subband, by applying GEnEI method and called Vertical Coefficient Gait Entropy Energy Image (VGEnEI). The last feature vector constructed from the first and second feature vector by first dividing human body into two-part Upper and Lower body part using on golden ratio proposition (Lower body $=0.32 \times$ height, Upper body $=0.62 \times$ height , and then for this feature in the upper body we use Vertical coefficient and for the lower body part we use Approximation coefficient, then the GEnEI applied for the created human body model. This feature called Approximation and Vertical Coefficients Gait Entropy Energy Image (AVGEnEI). Each mentioned feature vectors are tested separately and then fused based on decision level fusion.

In the third step k-Nearest Neighbor $(\mathrm{k}-\mathrm{NN})$ and SVM are applied separately as a classification method. To test the proposed method, we used CASIA $B$ gait database that contains 124 subjects (93 males and 31 females).

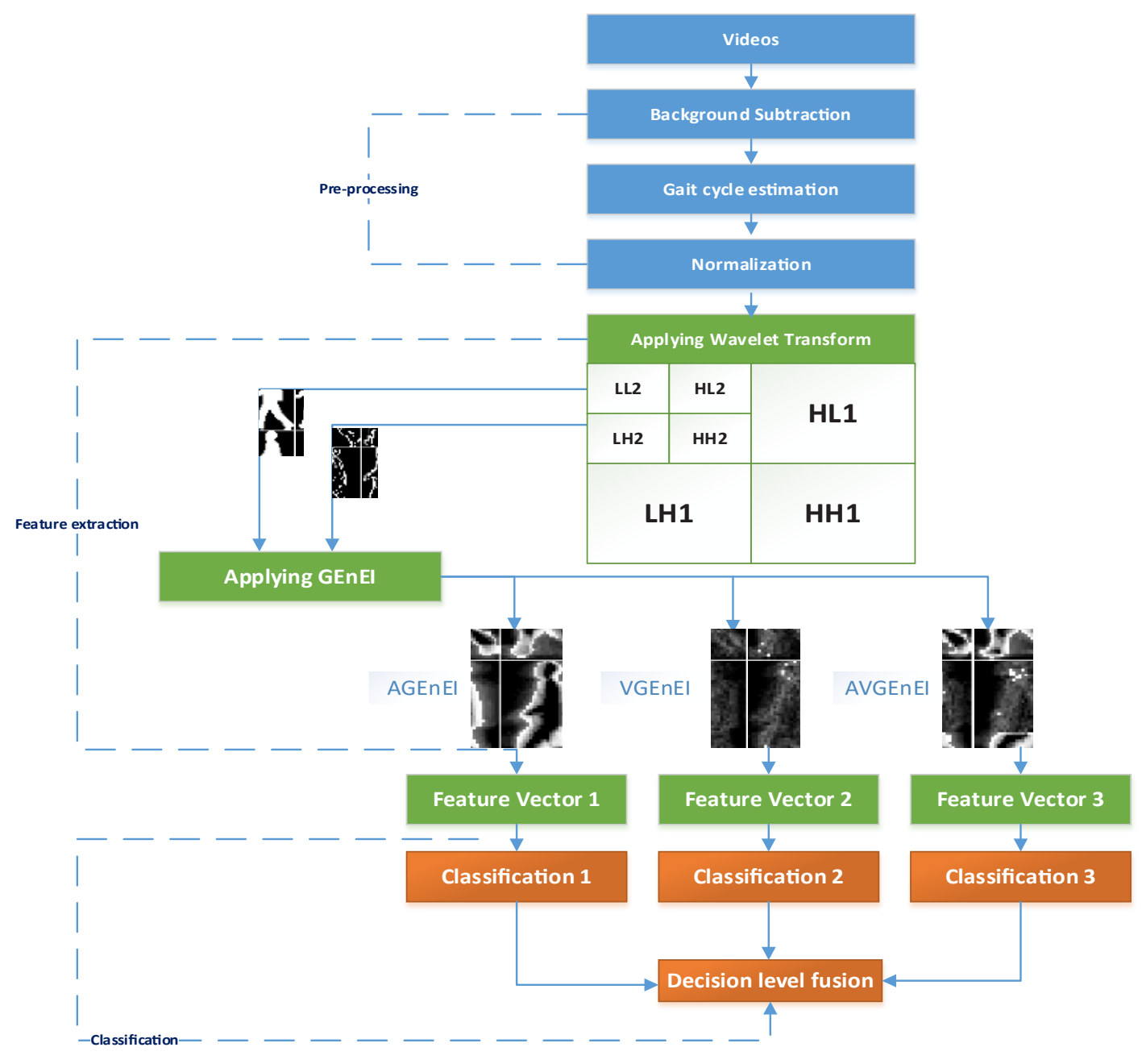

Figure 1. Overview of the proposed gender classification method 


\section{A. BACKGROUND SUBTRACTION}

This is a technique applied in the fields of image processing and computer vision. The background subtraction is used to extracted foreground image from the background image for further processing (human body recognition etc.). Background subtraction technique a widely utilized approach for detecting moving objects in videos from static cameras. Generally, regions of interest of an image are the objects (humans, cars, text etc.) in its foreground. Afterwards, object localization is required for which background subtraction algorithm is applied. With this technique, we can handle lighting changes, repetitive motions from clutter and long approach scene ${ }^{(17)}$

The following analyses make use of the function of $\mathrm{V}(\mathrm{x}, \mathrm{y}, \mathrm{t})$ as a video sequence where $\mathrm{t}$ is the time di-

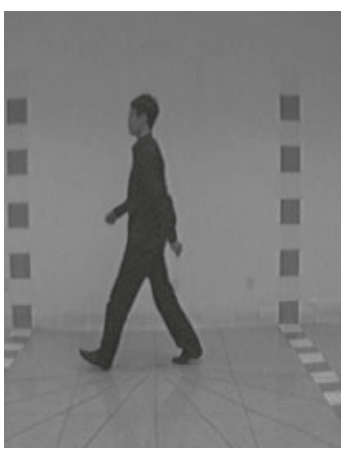

(a) mension, $\mathrm{x}$ and $\mathrm{y}$ are pixel area variables. e.g. $\mathrm{V}(1,2,3)$ will be those pixel intensities at $(1,2)$ pixel area of the picture during $t=3$ in the video sequence. Frame reference (absolute) at time $t+1$ is

$D(t+1)=|V(x, y, t+1)-V(x, y, t)|$

In this paper, we used frame difference method by calculating the difference between the consecutive frame and frame reference. Furthermore, a threshold is put on this difference image (D) to improve the subtraction. To generate the frame reference we calculate the average of the first ten frame assuming that there is no object on the scene. Box boundary, Normalization and alignment are applied for each of the silhouette images, this is followed by the gait cycle estimation as a final process in this step, see Figure 2.

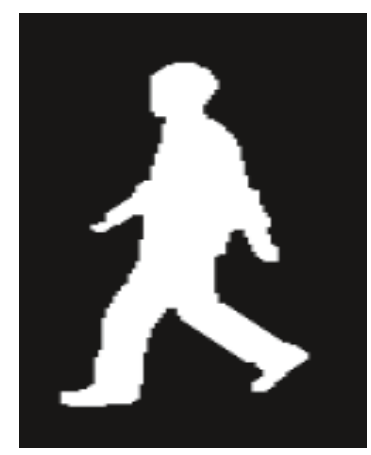

(b)

Figure 2. Process of background subtraction (a) Original image (b) Image after background subtraction.

\section{B. GAIT CYCLE ESTIMATION}

The gait cycle is described as the motion of the human frame from its initial placement at some supporting heels on the ground on the point when the same heel contacts the ground for the second time. A gait cycle is composed of one right leg step and one left leg step. The gait cycle is divided into two phases; stance, and swing. Stance is included in the interval in which the foot is on the ground, conversely Swing, and is included in the interval in which the foot is not in contact with the ground. The gait cycle of the motion is started when the heel at the stance phase of foot reaches to the ground and is finished when the heel reaches on the ground again in the next cycle. The distance between two heels is furthest at the start and finish stance positions. This procedure will collect $100 \%$ of the gait cycle. In order to robustly identify a person's gait signature, there is a need for the method for extracting gait cycles from a continuous signal of walking data. Commonly, there are two methods for estimating gait cycle. The first technique is the local minima, where both feet is nearby together. The second is the local maxima where the 
distance between the two feet are opened widely. In this paper, we used the double support phase (local

maxima) to determine gait cycle, see figure 3.

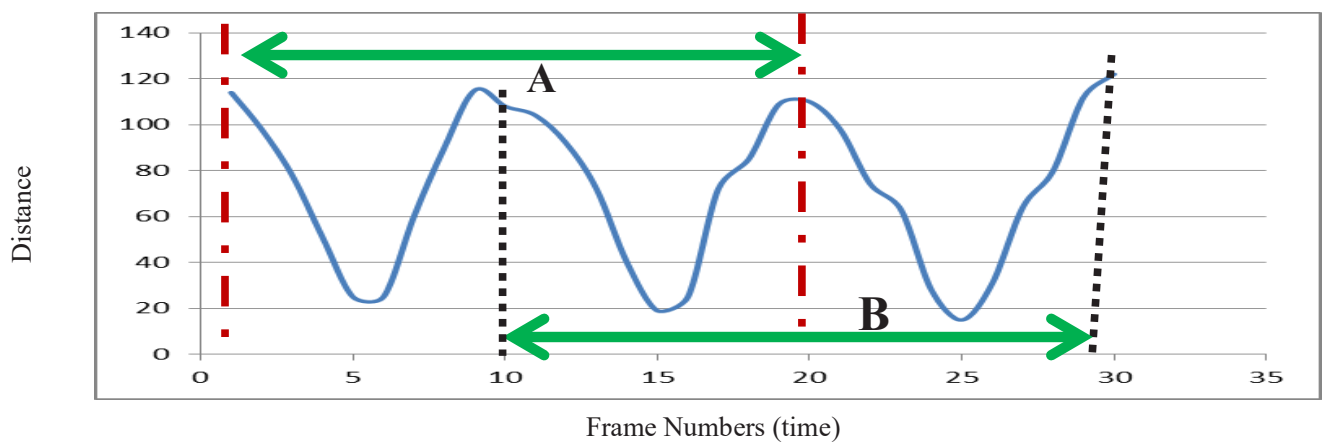

Figure 3. Gait cycle detection

\section{FEATURE EXTRACTION}

The process of extracting human gait signature (characteristics) called feature extraction, that will most efficiently or meaningfully represent the information for analysis and classification. In this paper, we extract a new feature based on two commonly used feature set GEI and GEnI, called GEnEI.

GEI and GEnI are two methods that are widely used in the literature as a gait feature, this is due to their simplicity and effectiveness. The limitation of GEI and GEnI is the lacking of robustness to deal with covariate conditions that affect the static areas of human body. Although GEnI is better than GEI in dealing with such problems, since GEnI reduces the effect of static parts that are influenced by bag and coat, however still both are not enough to provide good performance, especially in CW gait sequences case. Usually, each of GEI and GEnI is obtained from the spatial domain of the sequence of frames during one gait cycle. In this paper, we extract a new gait feature based on wavelet transform called Gait Entropy Energy Image (GEnEI). This feature is constructed based on GEI and GEnI. First, we compute GEI, then we check each pixel value in the constructed GEI, if the pixel value is between 0 and 0.5 , then we use the value of GEI in GEnEI, otherwise, we use the value of GEnI in GEnEI.
$\operatorname{GEI}(x, y)=\frac{1}{T} \sum_{t=1}^{T} I(\mathrm{x}, \mathrm{y}, \mathrm{t})$

$\operatorname{GEnI}(x, y)=-\sum_{\mathrm{k}=1}^{\mathrm{k}} \mathrm{p}_{\mathrm{k}}(\mathrm{x}, \mathrm{y}) \log _{2} \mathrm{p}_{\mathrm{k}}(\mathrm{x}, \mathrm{y})$

$\operatorname{GEnEI}(x, y)=\left\{\begin{array}{lr}\operatorname{GEI}(x, y) & \text { if } \operatorname{GEI}(x, y)>0 \text { and }<0.5 \\ \operatorname{GEnI}(x, y) & \text { Otherwise }\end{array}\right\}$

Where $\mathrm{T}$ is the number of frames in the sequence $I(x, y, t)$ is a binary silhouette image at frame $t ; x$, and $\mathrm{y}$ are the image coordinates. and $\mathrm{Pk}(\mathrm{x}, \mathrm{y})$ is the probability that the pixel takes on the $\mathrm{k}$-th value.

In this paper, three feature vectors are constructed from GEnEI using wavelet transformation method (level 2). The first feature vector is based on Approximation coefficient subband, called AGEnEI, the second feature vector is based on Vertical coefficient subband, called VGEnEI and the last feature vector called AVGEnEI. To construct the last feature, we divide the human body into two parts as upper and lower body, for the upper body part we use VGEnEI and for the lower body part we use AGEnEI, this is to reduce the effect of bags and coats that mostly affect the upper body part.

\section{EXPERIMENTAL SETUP}

We tested the performance of the proposed method by using the three feature vectors (AGEnEI, VGEnEI and AVGEnEI) separately and fusing them based on the decision level fusión. Decision-level fusion is the process of fusing the classification results of prior-classification stages. In this paper, the process is started by performing the classification results using each of the 3 feature sets separately, and then 
applying the majority voting method to perform the final decision. CASIA B Gait database is used to test the proposed method method, the database contains 124 participants (93 males and 31 females) and each participant has 6 samples. Due to imbalanced gender numbers in this database, we selected randomly equal subset of 25 males ( $25 * 6$ samples) and 25 females $\left(25^{*} 6\right.$ samples) of Neutral gait sequences $(\mathrm{Nu})$ to be used in the experiment. Now, we start to generate the gallery (training) and probe (test) set, in all experiments 10 fold cross-validation was applied to separate the gallery and probe set. The gallery set was constructed from the $\mathrm{Nu}$ gait sequence only, and the remaining records of $\mathrm{Nu}$, as well as the $\mathrm{CW}$ and $\mathrm{CB}$ gait sequences, are used as a probe set. These experiments are based on two different classification method (k-NN and SVM), that are used separately to test the performance of the proposed method. The experiment was repeated 30 times to give a chance for most of the $\mathrm{Nu}$ gait sequences to be used in the gallery and probe, see figure 4.

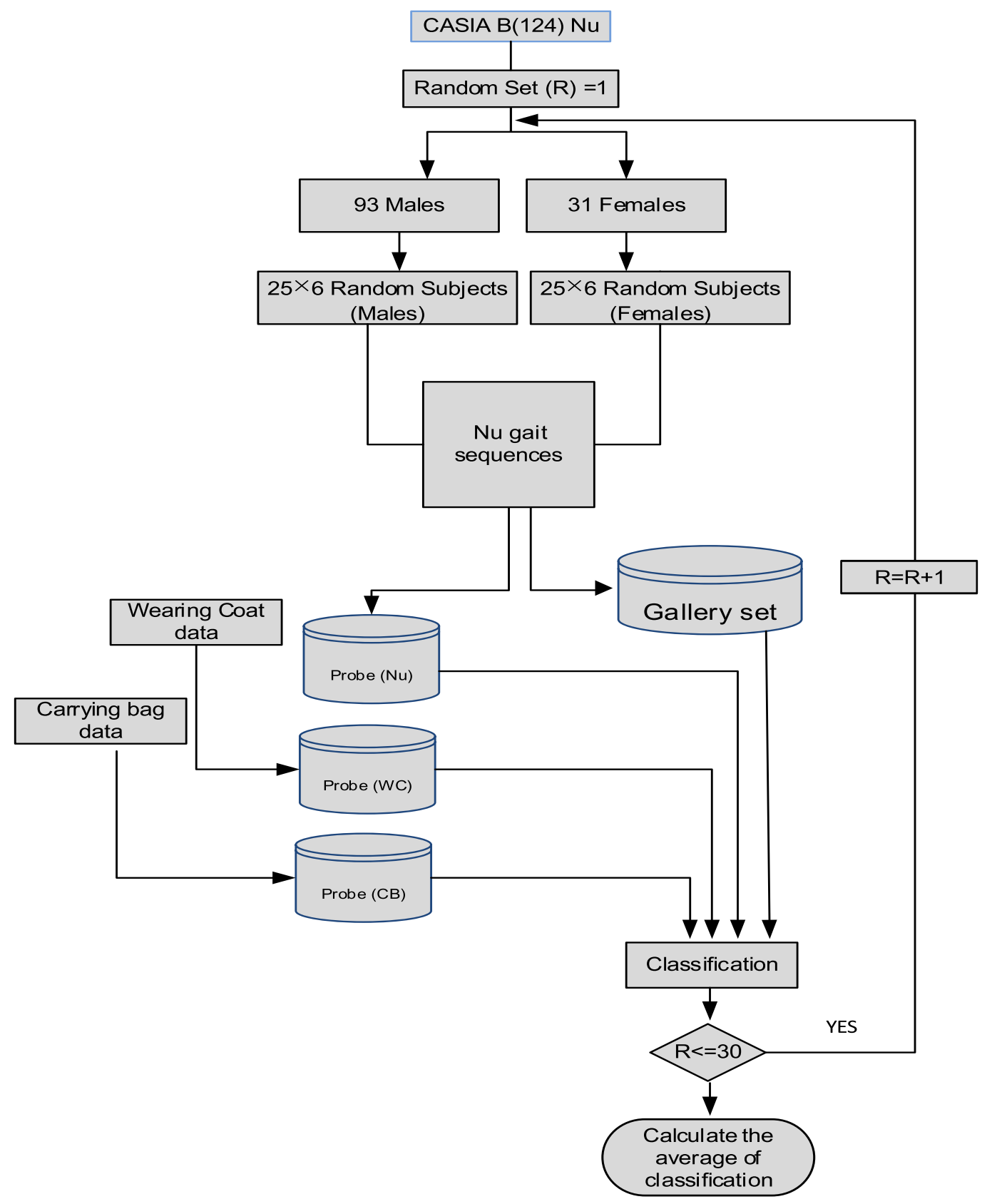

Figure 4. Generating gallery and probe set from the CASIA B for gender classification. 


\section{EXPERIMENTAL RESULTS AND DISCUSSION}

The experiments are divided into two parts; the first part of the experiment is based on $k-N N(k=1,3$, and 5), see Figure 5, 6 and 7. In this study, the average and standard deviation of the 30 repeated experiments is calculated and presented as a recognition accuracy. Figure 5, presents the result of k-NN with $\mathrm{k}=1$, the results show that for $\mathrm{Nu}$ gait sequence AGEnEI provided better result compared to VGE$\mathrm{nEI}$ and AVGEnEI, this is because approximation coefficient subband provide similar information as the original spatial image provided and also in this expermet the gallery and probe set contains $\mathrm{Nu}$ gait sequences.
In the case of using different gait sequence in the probe and gallery set, the experimental result shows that VGEnEI provided the better result for CB while for CW again AGEnEI provided better result compared to other set of feature. The reason behind these result is that the GEnEI originally reduced the effect of coat on the human body, that is why AGEnEI provided better result, while the effect of bag is still remain on the human body after using GEnEI, therefore VGEnEI which is based on the vertical subband provided better result compared to other two feature sets. When the three feature set are fused using decision level fusion, the accuracy significantly increased for all the cases, as shown in figure 5 .

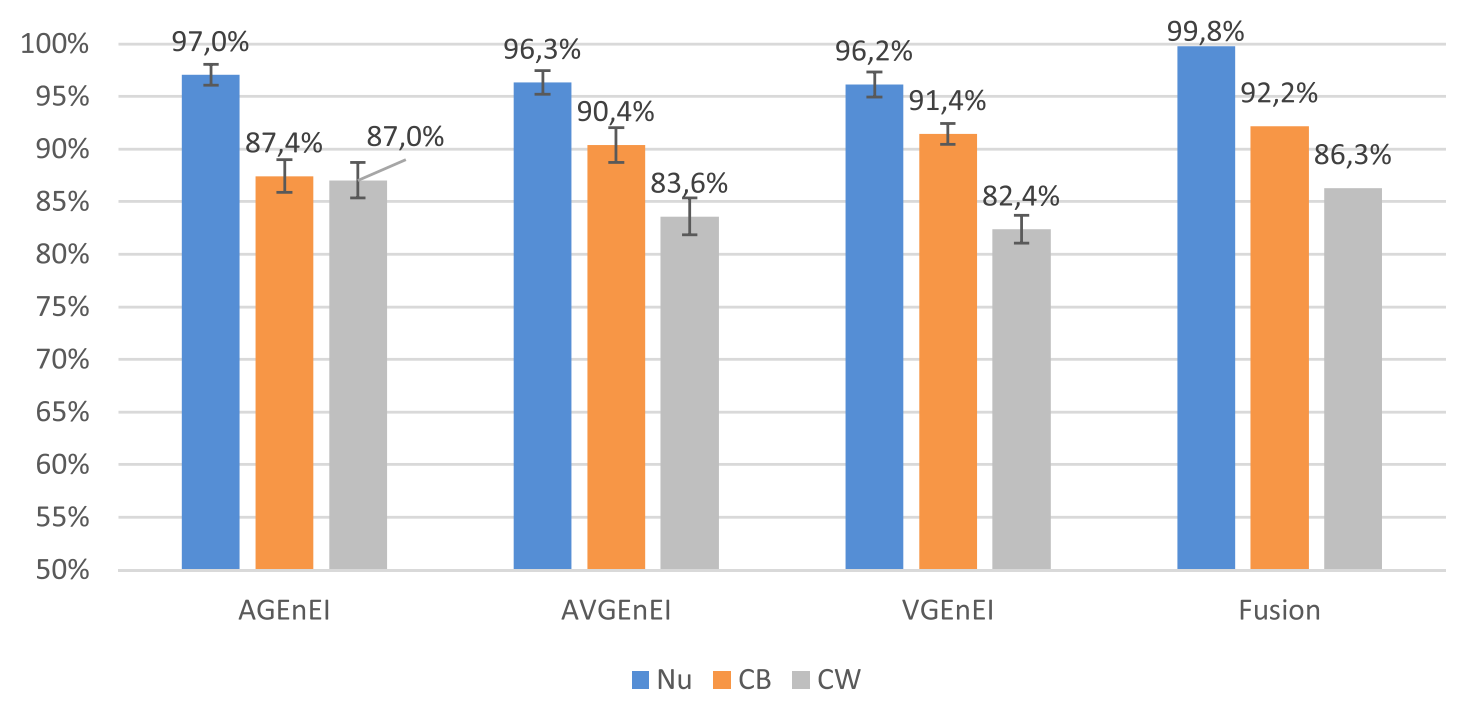

Figure 5. The results of the proposed method using $\mathrm{kNN}(\mathrm{k}=1)$, for each of $\mathrm{Nu}$ Normal, CB Carrying Bag and CW Coat Wearing.

In figure 6 we present the results using $\mathrm{k}-\mathrm{NN}$ with $\mathrm{k}=3$; the experimental result shows that for $\mathrm{Nu}$ and $\mathrm{CB}$ cases the results are below what provided by $(\mathrm{k}=1)$ in each of AGEnEI, VGEnEI and AVGEnEI with one exception (AGEnEI in CB case), while for $\mathrm{CW}$ the results are better than $(\mathrm{k}=1)$ in the case of using AVGEnEI and decision level fusion. 


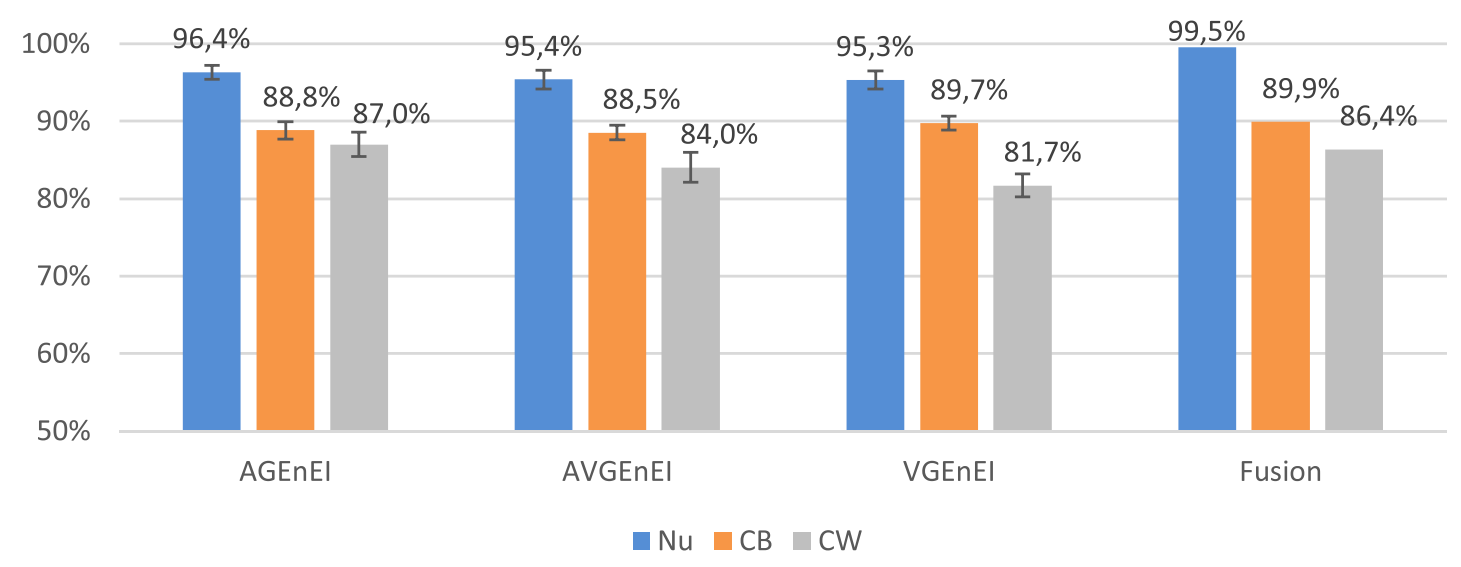

Figure 6. The results of the proposed method using k-NN (k=3), for each of Nu Normal, CB Carrying Bag and CW Coat Wearing.

Figure 7 , present the results of gender classification using $\mathrm{k}-\mathrm{NN}$ with $\mathrm{k}=5$, the experimental results show that in all of the case the results achieved using $\mathrm{k}=5$ are below what achieved by $k=1$ and $k=3$ with two exceptions (AGEnEI and VGEnEI in CW).

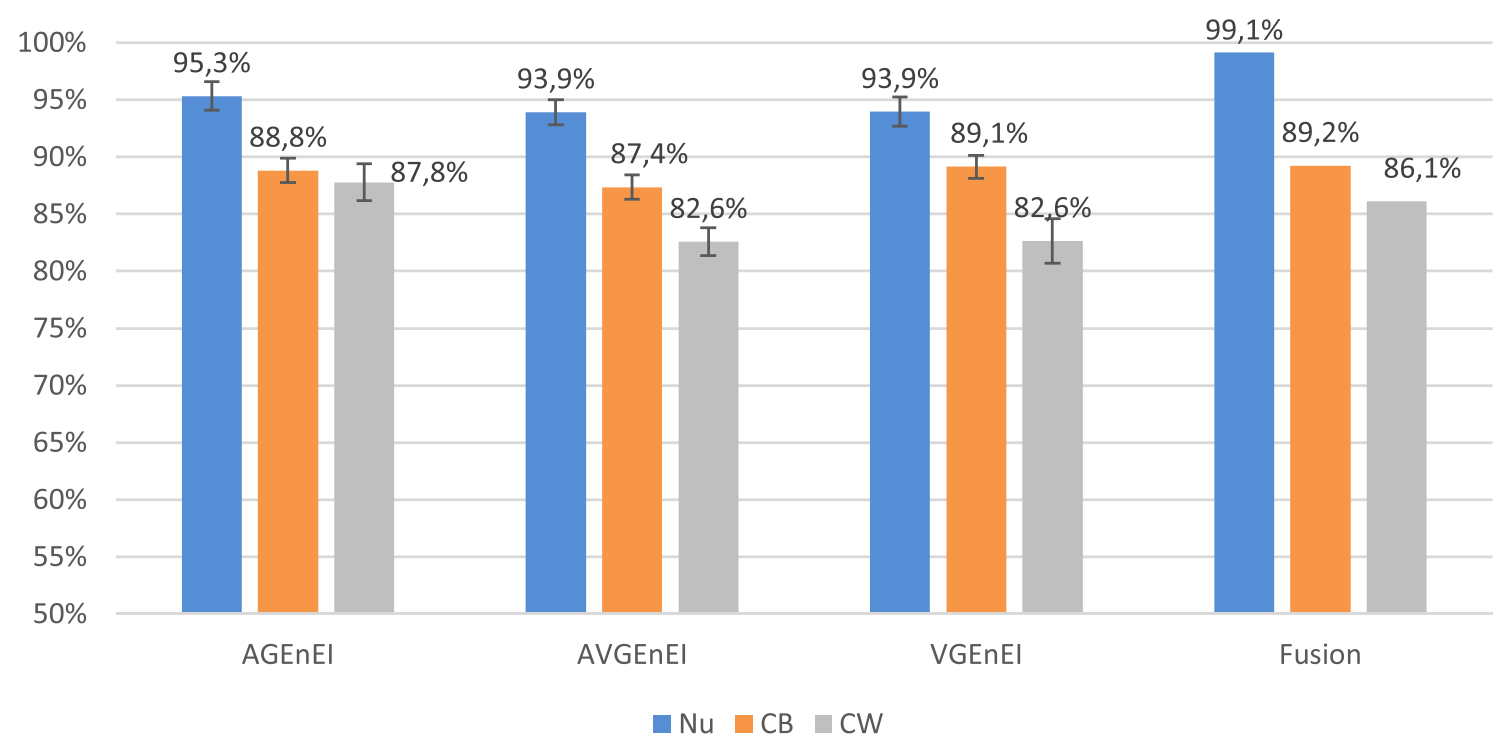

Figure 7. The results of the proposed method using $\mathrm{kNN}(\mathrm{k}=5)$, for each of $\mathrm{Nu}, \mathrm{CB}$ and $\mathrm{CW}$.

By looking at the results provided using (k-NN with $\mathrm{k}=1,3$, and 5), we conclude that $\mathrm{k}=1$ provide relatively better accuracy compared to $\mathrm{k}=3$ and 5 , and the results performed based on the decision level fusion is better than using each of the feature set separately in all of the cases. In the next experiment, we shall test the performance of the proposed method based on SVM using the same feature sets that used in the previous experiment, (see Figure 4.6).

First, each of the features set tested separately, then decision level fusion is used to combine the three feature sets (AGEnEI, VGEnEI, and AVGEnEI). Experimental results show that in general, $\mathrm{Nu}$ gait 
sequences provided better results compared to $\mathrm{CB}$ and CW. For Nu case, AGEnEI provided 97.3\%, which is better than VGEnEI and AVGEnEI that provided $96.7 \%$ and $94.4 \%$ respectively. For CB the results are below what the method achieved by $\mathrm{Nu}$ case, this is because of two reasons; first, the gallery set contains $\mathrm{Nu}$ gait sequences only, secondly due to the effect of bags on the human body. In term of the feature set, AGEnEI and AVGEnEI provided $66.7 \%$ and $66.3 \%$ respectively, while in VGEnEI the recognition rate is $78.9 \%$ which is better com- pared to other two sets, this is because VGEnEI is based on the vertical coefficients only. In the case of $\mathrm{CW}$ the result is relatively better than $\mathrm{CB}$ for each AGEnEI, AVGEnEI, and VGEnEI which provides $76.2 \%, 81.2 \%$ and $83 \%$ respectively.

The decision level fusion in this experiments leds to increase the result in $\mathrm{Nu}$ gait sequences, while for $\mathrm{CB}$ and $\mathrm{CW}$ the results are below what achieved by VGEnEI.

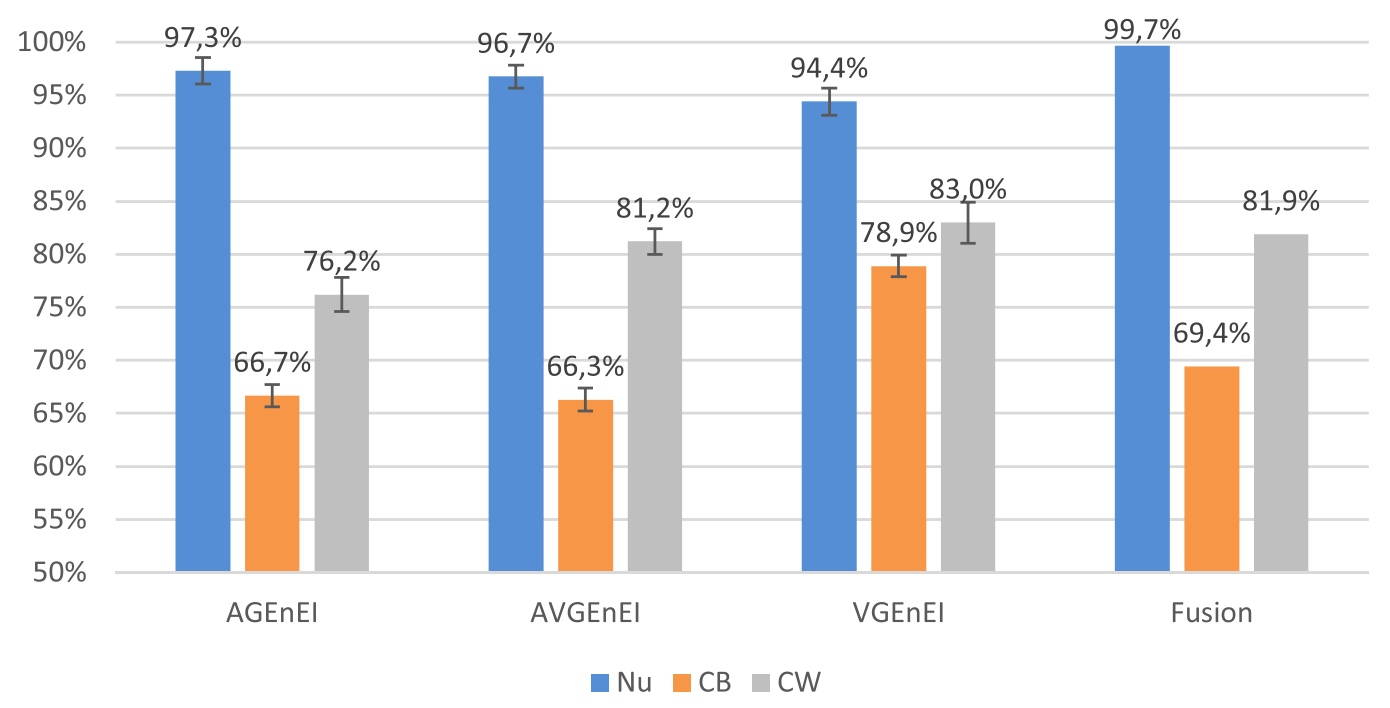

Figure 8. The results of the proposed method using SVM, for each of Nu normal, CB Carrying Bag and CW Coat Wearing.

To preset the effect of the classification methods in the proposed scheme we shall compare the use of $\mathrm{k}-\mathrm{NN}$ and SVM. Table 1, present the results achieved by SVM and k-NN using each of the feature set and their fusion. The experimental results show that for $\mathrm{Nu}$ gait sequences SVM provide a better result for each of AGEnEI and AVGEnEI, while in VGEnEI and decision level fusion, $\mathrm{k}-\mathrm{NN}$ with $\mathrm{k}=1$ is provided better accuracy. For $\mathrm{CB}$ and $\mathrm{CW}$ gait sequences $\mathrm{k}-\mathrm{NN}(\mathrm{k}=1)$ provided better results compared to
SVM for all the cases with one exception (VGE$\mathrm{nEI}$ in $\mathrm{CW}$ ). The reason behind the results of $\mathrm{Nu}$ is that, for $\mathrm{Nu}$ gait sequences the gallery and probe set contain the same type of gait sequences (Nu gait sequences), thus in VGEnEI some necessary information are removed, due to using vertical subband only, while in $\mathrm{CB}$ and $\mathrm{CW}$ the galley and probe contain different gait sequences, thus bags and coat have an effect on the results negatively. 
Table 1. Comparitsion results of the proposed methods using $\mathrm{kNN}(\mathrm{k}=1)$ and SVM

\begin{tabular}{ccccc}
\hline k-NN (k=1) & AGEnEI & AVGEnEI & VGEnEI & Fusion \\
\hline $\mathrm{Nu}$ & $97.0 \%$ & $96.3 \%$ & $96.2 \%$ & $99.8 \%$ \\
$\mathrm{CB}$ & $87.4 \%$ & $90.4 \%$ & $91.4 \%$ & $92.2 \%$ \\
$\mathrm{CW}$ & $87.0 \%$ & $83.6 \%$ & $82.4 \%$ & $86.3 \%$ \\
\hline SVM & & & \\
\hline $\mathrm{Nu}$ & $97.3 \%$ & $96.7 \%$ & $94.4 \%$ & $99.7 \%$ \\
$\mathrm{CB}$ & $66.7 \%$ & $66.3 \%$ & $78.9 \%$ & $69.4 \%$ \\
$\mathrm{CW}$ & $76.2 \%$ & $81.2 \%$ & $83.0 \%$ & $81.9 \%$ \\
\hline
\end{tabular}

Now, we shall compare our result with the published work in the literature. In the process of comparison, we selected those methods that proposed gender classification in the neutral and non-neutral case and used CASIA B gait database. The comparison shows that our scheme outperforms the two other schemes with one exception (CW case in $\stackrel{(16)}{)}$.

Table 2. Comparing propoed method with results published in the literature

\begin{tabular}{ccccc}
\hline Methods & Nu \% & CB \% & CW \% & Average of (Nu, CB and CW) \% \\
\hline (15) & 96.7 & 88.7 & 83.8 & 89.8 \\
(16) & 97.6 & 90 & 87.5 & 91.7 \\
Proposed Method & 99.8 & 92.2 & 86.3 & 92.8 \\
\hline
\end{tabular}

\section{CONCLUSIONS}

In this paper we investigated gait-based gender classification method using Neutral $(\mathrm{Nu})$ and Non-neutral (Carrying Bags (CB) and Coat Wearing (CW)) gait sequences. A new feature set is used that generated from GEnI and GEI and called GEnEI. This feature has been presented in three different form based on wavelet transform, using approximation and non-approximation coefficient called (AGEnEI,
VGEnEI and AVGEnEI). To test the performance of the proposed method, CASIA B gait database used as a large and widely used database. Two different classification method is used separately called $\mathrm{k}-\mathrm{NN}$ and SVM. In the proposed method the three mentioned feature sets were tested separately and fused using decision level fusion. As an addition to $\mathrm{Nu}, \mathrm{CB}$ and $\mathrm{CW}$ gait sequences were used as well to address the external factor of gait challenges. The gallery set consists of a part of $\mathrm{Nu}$ gait sequences 
only, while in the probe set we test the remaining $\mathrm{Nu}$ as well as $\mathrm{CB}$ and $\mathrm{CW}$ gait sequences. The experimental result indicates that in the case of using $\mathrm{k}-\mathrm{NN}$ as a classification method $\mathrm{k}=1$ provided better result compared to $\mathrm{k}=3$ and $\mathrm{k}=5$. When the method tested based on SVM the experiments show that for $\mathrm{Nu}$ case, AGEnEI and AVGEnEI provided 97.3\% and 96.7 respectively, these results are better than $97 \%$ and $96.3 \%$ which provided by the same two feature set using k-NN. However, SVM outperformed by k-NN using VGEnEI feature set by $1.8 \%$.

Moreover, for $\mathrm{CB}$ and $\mathrm{CW}$ gait sequences, k-NN $(k=1)$ provided better accuracy than SVM except in the case of using VGEnEI for CW, in which SVM outperform $\mathrm{k}-\mathrm{NN}$ by $0.6 \%$. The reason behind these results is that SVM is supervised and k-NN is unsupervised classification method. Combining the three proposed feature sets (AGEnEI, VGEnEI and AVGEnEI) using $\mathrm{kNN}$ at the Decision level fusion has led to highest accuracy in comparison with using each feature set separately as well as the state of the art. Furthermore, decision level fusion based on SVM has not improved the result except in the case of $\mathrm{Nu}$ case. Although in general, k-NN provides better results with low dimensional space and SVM does with high dimensional space, however, the performance of both classifiers initially depends on the dataset chosen.

Few research works, including the current paper, used side view angle for gait-based gender classification. In real time scenarios, you can not guarantee human gait with specific view angle (direction), in future work we will focus on investigating different view angle for gender classification.

\section{REFERENCES}

1. Choudhary J. Survey of Different Biometrics Techniques. International Journal of Modern Engineering Research (IJMER). 2012;: p. 2249$-6645$.

2. Liu , Ye, Li , Zhang F, Lin L. Memory-based Gait Recognition. In BMVC.; 2016.

https://doi.org/10.5244/C.30.82

3. Sabir, Al-jawad, Jassim S. Gait recognition using spatio-temporal silhouette-based features. In Mobile Multimedia/Image Processing, Security, and Applications 2013 \}; 2013; Baltimore, Maryland, United States.

https://doi.org/10.1117/12.2017950

4. Sabir, Ahmed H, Faeq K, Maghdid S. Human gait identification using Kinect sensor. Kurdistan Journal of Applied Research. 2017; 2: p. 142--146. https://doi.org/10.24017/science.2017.3.37

5. Ahmed H, Sabir T. Human Gender Classification Based on Gait Features Using Kinect Sensor. In 3rd IEEE International Conference on Cybernetics (CYBCONF); 2017 ; Exeter, UK.

https://doi.org/10.1109/CYBConf.2017.7985782

6. Mather, Murdoch L. Gender discrimination in biological motion displays based on dynamic cues. Proc. R. Soc. Lond. B. 1994; 258: p. 273-279. https://doi.org/10.1098/rspb.1994.0173

7. Kozlowski LT, Cutting JE. Recognizing the sex of a walker from a dynamic point-light display. Perception $\backslash \&$ psychophysics. 1977; 21(6): p. 575-580.https://doi.org/10.3758/BF03198740

8. Kastaniotis D, Theodorakopoulos I, Economou GaFS. Gait-based gender recognition using pose information for real time applications. In 2013 18th International Conference on Digital Signal Processing (DSP).: IEEE; 2013.

https://doi.org/10.1109/ICDSP.2013.6622766

9. Andersson VO, Amaral LS, Tonini AR, Araujo RM. Gender and body mass index classification using a microsoft kinect sensor. In The Twenty-Eighth International Flairs Conference; 2015. 
10. Gianaria E, Grangetto M, Lucenteforte M, Balossino N. Human classification using gait features. In International workshop on biometric authentication, Springer; 2014. p. 16-27. https://doi.org/10.1007/978-3-319-13386-7_2

11. Lee, Grimson WEL. Gait analysis for recognition and classification. In Fifth IEEE International Conference on Automatic Face and Gesture Recognition, 2002 ; 155--162; Washington, DC, USA: Automatic Face and Gesture Recognition, 2002. Proceedings. Fifth IEEE International Conference on.

12. Wang L. Behavioral Biometrics For Human identification:Intelligent Application: Intelligent Applications. IGI Global,; Aug 31, 2009. https://doi.org/10.4018/978-1-60566-725-6

13. Yu , Tan, Huang, Jia , Wu X. A study on gaitbased gender classification. IEEE Transactions on image processing. 2009; 18(8): p. 1905-1910. https://doi.org/10.1109/TIP.2009.2020535
14. Jalil SZA, Taib MN, Abdullah H, Yunus MM. Frequency Radiation Characteristic Around The Human Body. International Journal of Simulation: Systems, Science \\& Technology. 2011; 12(1): p. 34--39. https://doi.org/10.1109/ICPR.2010.897

15. Hu M, Wang, Zhang Z, Wang Y. Combining spatial and temporal information for gait based gender classification. In 20th International Conference on Pattern Recognition (ICPR); 2010 ; Istanbul, Turkey.

16. Sabir, Al-Jawad, Jassim S. Feature selection gaitbased gender classification under different circumstances. In Proc. Real-Time Image and Video Processing, SPIE. ; 2014; Brussels, Belgium. https://doi.org/10.1117/12.2052586

17. Piccardi M. Background subtraction techniques: a review. In 2004 IEEE International Conference on Systems, Man and Cybernetics (IEEE Cat. No. 04CH37583; 2004: IEEE International Conference on Systems. p. 3099--3104. 